\title{
LA ESCASEZ DE ENERGÍA ELÉCTRICA EN LA POSTGUERRA (1943-50) Su repercusión en la economía alicantina*
}

\author{
ROQUE MORENO FONSERET \\ Universidad de Alicante
}

Los intentos de desarrollo industrial que vivió España tras la Guerra Civil tropezaron a lo largo de la década de los cuarenta con la escasez de materias primas, de bienes de equipo y de energía. Los cupos para practicamente todas las materias primas (incluidas en ocasiones las nacionales), las licencias de importación y la carencia de divisas unido a las restricciones de energía eléctrica, fueron circustancias comunes en ese decenio que provocaron el estancamiento económico general de España, tal como han puesto de manifiesto todos los estudios científicos sobre el tema. (1)

Quizás de los tres factores citados sea el último (escasez de energía eléctrica) el menos tratado y, sin embargo, en determinados años constituyó la causa fundamental de recesión económica llegando a paralizar las fábricas cuatro días a la semana con el consiguiente descenso en la producción. (2)

El presente trabajo constituye un intento de aproximación a la incidencia que las restricciones eléctricas tuvieron sobre la economía alicantina, más atec-

* En este artículo se adelantan algunos resultados de la tesis que sobre la economía en la provincia de Alicante durante el primer franquismo realiza el autor bajo la dirección de don Salvador Forner Muñoz, mediante el disfrute de una beca de investigación concedida por la Caja de Ahorros de Valencia.

(1) Desde el ya clásico estudio de J. Clavera y otros, Capitalismo español: de la autarquía a la estabilización (1939-1959), Madrid, 1978, a otros de esta década como R. Carr, España 1808-1975, Barcelona, 1984; J. Fontana y J. Nadal, «España 1914-1970» en C.M. Cipolla ed., Historia económica de Europa. Economías contemporáneas, Barcelona, 1980; M. J. González, La economía política del franquismo (1940-1970), Madrid, 1979. Una buena visión del estado historiográfico del tema aparece en J. L. Garcia Delgado, «Estancamiento industrial e intervencionismo económico durante el primer franquismo» en J. Fontana ed., España bajo el franquismo, Barcelona, 1986.

(2) El año de mayor carestía eléctrica fue el de 1945. En 1960 se celebra la Conferencia Mundial de la Energía, calculando las ponencias presentadas sobre el tema que nos ocupa («Las restricciones eléctricas y su repercusión en la economía española», ponencia I B/15 presentada por M. Gallardo, J. Iribarren, F. Prieto, F. Barcelo y J. M. Trejo, e «lncidencia de las restricciones eléctricas sobre la economía nacional», ponencia I B/11 presentada por José Castañeda y J.L. Redonet) que las restricciones en 1945 causaron perjuicios por valor del 14 por 100 de la renta nacional y del 40 por 100 de la renta industrial, tal como recoge R. Tamames, Estructura económica de España, Madrid, 1982. 
tada que la nacional, analizando, por un lado, los perjuicios en el campo y en la industria y, por otro, las consecuencias sobre el obrero industrial que ve reducir su salario en proporción directa al aumento de las horas de corte de fluido. Si bien las restricciones comienzan en 1943 y alcanzan hasta 1955, nos centraremos en la década de los cuarenta, período en el que los cortes son mayores y van acompañados de la impotencia del Gobierno para solucionar el problema.

\section{LAS RESTRICCIONES ELÉCTRICAS}

Los cortes eléctricos que comenzó a sufrir España en 1943 tienen su origen fundamentalmente en el aumento importante del consumo de energía (3). Dicho ascenso vino a producirse en unos momentos en que existían graves dificultades para satisfacerlo derivadas de la Segunda Guerra Mundial que imposibilitaba la importación de materias primas de las que se carecía y que eran necesarias para la conducción eléctrica, como cobre y aluminio, o de bienes de equipo para las centrales. El posterior aislamiento de España no hizo sino confirmar la solución autárquica pretendida, que para el caso de la electrificación nacional queda claramente reflejada en las palabras del Ministro de Obras Públicas, A. Peña Boeuf, el cual señalaba en 1944:

«La electrificación está supeditada ... a la construcción de máquinas, aparatos y utillaje preciso para realizar las instalaciones ... (y esto es) de dificil y lenta solución. Tengase en cuenta que España no contaba con una industria preparada para atender a estas necesidades. Las experiencias hechas con motivo de los trabajos de electrificación realizados hasta hoy darán sus frutos y en un porvenir no lejano podrán alcanzar nuestras factorías la capacidad precisa para poder atender la demanda de material que necesitamos, se habrá llegado asimismo a contar con los obreros especializados en número suficiente e incluso con un cuadro de técnicos bastante bien preparados» (4)

(3) La producción eléctrica en España durante 1931 fue de 2.681 millones de Kwh y en 1935 fue de 3,271 millones, lo que supone un incremento anual de 5,5 por 100 . Entre 1940 (3.617 millones Kwh) y 1950 (8.299 millones kwh) el incremento anual es de 10,1 por 100. El desequilibrio entre la producción de energía y el consumo es claro si tenemos en cuenta que la producción nacional en 1942 fue de 4.400 miliones de kwh y las necesidades contratadas excedian en mucho de los 6.000 millones de kwh, según estudio presentado al Consejo Económico Sindical Provincial en 1946 por el Ingeniero Jefe de la provincia de Alicante (Archivo Histórico Provincial de Alicante, sección AISS, Legajo 2332-3a, Carpeta $4 .^{a}$ ).

(4) Información, 27 de diciembre de 1944. 
El autoabastecimiento pasaba pues por un proceso de aprendizaje empírico que hacía «muy arriesgado el calcular cuando puede estar concluida la electrificación total proyectada" y que reflejaba en resumidas cuentas soluciones "que podemos calificar de improvisadas» según palabras del propio ministro. Si a estos deseos autárquicos añadimos la falta de divisas para hacer frente a los gastos de los costosos bienes de equipo obtendremos un cuadro para la industria eléctrica caracterizado por un desarrollo muy lento.

La intervención estatal, en concreto, se manifestó fundamentalmente en un importante plan de construcción de embalses cuya finalidad principal era la puesta en riego de amplias zonas de secano, si bien sus saltos a pie de presa eran concedidos por el Ministerio de Obras Públicas a las sociedades eléctricas para la construcción de centrales. Sin embargo, el retraso en la construcción fue grande debido, por un lado, a la escasez de materiales ya mencionada y, por otro, al bloqueo de tarifas (5).

La obstinación en mantener fijas las tarifas supuso el mayor freno para el desarrollo eléctrico español y ello a pesar de las continuas quejas de las empresas eléctricas. Ya en 1943 el Sindicato de Agua, Gas y Electricidad de Alicante señalaba en el bloqueo la causa principal de la lentitud en la electrificación nacional:

«Este problema (falta de energía) podría solucionarse con la instalación de nuevas centrales ... gastos todos ellos que las Compañias de Electricidad no pueden hacer frente porque las tarifas de venta de fluido eléctrico que rigen actualmente son las mismas que las de antes del Glorioso Movimiento Nacional en 1936. Todas las tarifas de servicios públicos como ferrocarriles, tranvias, etc, han sido aumentadas para hacer frente a la nueva situación; en cambio, las tarifas de energía eléctrica rigen las mismas del año 1936, a pesar de ello las Compañías de Electricidad han aumentado el sueldo a sus productores ... pero están retraidas en la inversión de nuevo capital para la construcción de nuevas centrales.» (6)

Efectivamente, las empresas presentaron cierta resistencia a realizar nue-

(5) R. Tamames, op. cit. Las tarifas fijadas antes de la guerra quedaron casi totalmente bloqueadas hasta el 1 de enero de 1953, fecha en la que entró en vigor el régimen de Tarifas Tope Unificadas (TTU).

(6) Archivo Histórico Provincial de Alicante, Sección AISS, Legajo 2332-3a, Carpeta 6. ${ }^{\text {a }}$. Se trata del resumen de las tareas desarrolladas por el I Consejo Provincial de Ordenación Económica. (en adelante AHPA). 
vas construcciones ante la prohibición oficial de aumentar las tarifas. El Estado, no obstante, tras la escasez de 1945 exigió de las Compañías eléctricas la inmediata construcción de las centrales amenazando incluso con hacer caducar las concesiones (7). Las lamentaciones lógicamente continuaron (8) y a pesar de que se reconocía que la subida de tarifas era la única solución viable no se hizo nada al respecto en la década de los cuarenta. En 1946, el Consejo de Industria señalaba que los trabajos de construcción implicaban gastos que no podían realizar las empresas sin la contrapartida necesaria de la revisión de tarifas, si bien no se llevaba a cabo debido a que era un «asunto complejo, porque para alcanzar soluciones justas precisa establecer como pùnto de partida consideraciones de muy distinta naturaleza» (9)

Fa ltaba pues una política de ordenación de la industria eléctrica que permitiese resolver el problema de la falta de energía que frenaba el desarrollo económico español (10); política que no se realizó hasta bien entrado los años cincuenta autorizándose ciertos aumentos de precios y concediéndose una subvención de parte de las tarifas cobradas como prima a las nuevas construcciones (O.F.I.L.E.). Podemos señalar por tanto que la raiz de la escasez se centraba en la indiferencia estatal que sólo se preocupaba del problema cuando cíclicamente éste se veía agravado por la falta de precipitaciones que,

(7) S. Llobet, "La energía eléctrica en España» en Estudios Geográficos, número 70, Madrid, 1958.

(8) En 1946 en el estudio presentado al I Consejo Económico Sindical Provincial de. Alicante ya citado, el Ingeniero Jefe decía que «a explotación de nuevos saltos de agua no resulta económicamente remuneradora de no aumentarse -al decir de las empresas- las tarifas. Las nuevas instalaciones eléctricas son, en la actualidad, carísimas y sus gastos de primer establecimiento no pueden en modo alguno cubrirse con los recursos que proporcionan las actuales tarifas».

(9) Ministerio de Industria y Comercio, Estado actual de la producción y distribución de la energía eléctrica en España 1945, Madrid, 1946.

(10) El Consejo de Industria en 1946 proponia:

- Terminación de las nuevas centrales generadoras hidráulicas y de los embalses reguladores en construcción, asi como el montaje de las centrales y estímulo reguladoras en proyecto, adoptando para ello medidas de protección y estímulo.

- Estimular a las empresas para mejorar el rendimiento de las actuales instalaciones, subsanando deficiencias tales como la insuficiencia de sección de algunas líneas de transporte y redes distribuidoras y ciertos trasiegos de energia evitables.

- Continuación de la política de construcción de centrales y supercentrales.

- Condicionar la concesión de nuevas instalaciones hidroeléctricas a la creación en el sistema de las proporcionales reservas térmicas.

- Creación de una red eléctrica nacional mediante interconexiones que eliminen los grandes islotes desconectados del sistema como la Andalucia occidental.

- Severa represión del fraude.

vid. Ministerio de Industria y Comercio, op. cit. 
en modo alguno, debe entenderse como causa básica de las restricciones. La «pertinaz» sequía de 1944-5 influyó grandemente en el descenso de la producción de esos años y en menor medida lo hizo la de 1948, pero el problema era estructural y su solución no pasaba por un aumento de las lluvias (11), a pesar de que en ocasiones se achacaran a la falta de ellas los cortes, sobre todo cuando fuera de España se comenzaba a atacar al Gobierno como incapaz de resolver la escasez energética. En 1945 el nuevo Ministro de Obras Públicas, Fernández Ladreda, señalaba:

"Las adversas circunstancias de la excepcional sequía que sufre nuestro país han sido utilizadas por los enemigos de España en el exterior como plataforma de los injustos y reiterados ataques. Ante la agravación que en algunos aspectos ha adquirido ritmo vertiginoso de dichas circunstancias (restricciones) motivadas por la persistencia de una sequía que bien puede calificarse de calamidad nacional, parece oportuno informar al país ... no sólo con el ánimo de salir al paso de los mencionados ataques ... sino para hacer un llamamiento a la serenidad y patriotismo» (12).

\section{LAS RESTRICCIONES EN LA PROVINCIA DE ALICANTE}

Los cortes de fluido eléctrico no afectaron por igual a todas las regiones españolas. El Ministerio de Industria y Comercio a través de la Dirección General de Industria dedicó especial atención a reservar las escasas disponibilidades para aquellos sectores de consumo de mayor interés en la economía nacional (industrias de base fundamentalmente) (13). La provincia de Alicante, con un importante peso específico dentro de las industrias de transformación (calzado y textil), no disponía en cambio de factorías incluidas en el grupo favorecido y sufrió cortes más intensos que el conjunto de la economía

(11) Ello era reconocido por las autoridades del momento. A. Peña Boeuf, Ministro de Obras Públicas, señalaba que «la causa de la escasez de fluido eléctrico padecida en el año actual (1944) y en el pasado no es ólo la penuria de agua... además ha influido de modo tan importante por lo menos el extraordinario aumento del consumon en Información, 27 de diciembre de 1944. El Ingeniero Jefe de la Delegación de Industria de Alicante respondía que ula lluvia alivia indudablemente pero no resuelve la crisis de producción. Mientras ésta exista es necesario el sistema de restricciones» en Información, 24 de octubre de 1944.

(12) Información, 17 de octubre de 1945.

(13) vid. por ejemplo la Orden de la Presidencia del Gobierno de 26 de septiembre de 1945 por la que se restringen las actividades laborales con motivo de la escasez de energía eléctrica, en la que se especifica que las preferencias en tiempo y cuantía de las restricciones se efectuarán a favor de las entidades de mayor interés nacional, que pueden quedar incluso exentas de restricción. Boletín Oficial de la Provincia de Alicante, 3 de octubre de 1945 (en adelante B.O.P.) 
nacional. Por ello no es extraño encontrar en las Disposiciones sobre restricciones eléctricas elaboradas por el Delegado Técnico de la zona CentroLevante como causa de las restricciones «el tener que enviar energías a las zonas del Norte para atenciones nacionales primordiales" (14).

Las diferencias y privilegios no existían sólo entre las diversas regiones energéticas de España, sino que dentro de la misma zona las había en función de la importancia de la actividad económica desarrollada. Así por ejemplo, por necesidades ineludibles de Altos Hornos de Vizcaya en Sagunto (Valencia), a los que se precisaba suministrar corriente de buenas características de 12 a 2 de la tarde, se hacía necesario el paro del 50 por 100 de la industria durante dichas horas en el resto de la zona levantina. Por otra parte, Madrid no tuvo un régimen de restricciones tan severo como el resto de las provincias de la zona. En el informe emitido por la Delegación Provincial de Madrid en 1945 se puede leer:

«Las restricciones conseguidas por las empresas de esta provincia no fueron tan elevadas como las que correspondieron a algunas situadas en otras provincias de la zona de Levante que se regian por las mismas normas» (15).

La raíz del problema se centraba fundamentalmente en el carácter importador de energía eléctrica de la provincia de Alicante con medios propios de producción poco importantes en relación con sus necesidades. La producción de electricidad en la provincia, que tal como refleja el cuadro 2 representaba aproximadamente el 10 por ciento del consumo provincial, se realizaba en la Central Hidráulica de Almadenes de la Compañía Riegos de Levante (situada en Murcia) y las centrales térmicas de la misma Compañía situadas en Alicante capital y Crevillente. Fuera de estas centrales no existen otras fuentes de producción de relativa importancia, teniendo que abastecerse de los saltos de agua situados en la zona de Levante explotados por la Compañía Hidroeléctrica Española con los que se suministra a las provincias de Castellón, Valencia, Murcia, Alicante y Madrid. (16)

El abastecimiento, tanto de la energía propia como de la importada, no estaba asegurado. En cuanto a la producción provincial, la Central de Almadenes no disponía de más caudal de agua que el procedente de los desem-

(14) B.O.P., 14 de octubre de 1946.

(15) Ministerio de Industria y Comercio, op. cit.

(16) Cámara Oficial de Industria, Comercio y Navegación de Alicante, Memoria Comercial 1944-5, Alicante, 1946. (en adelante COCINA). 
balses de los pantanos de Fuensanta y Talava, regidos por la «Confederación de Riegos del Segura", residente en Murcia, la que acomodaba sus aportaciones de agua atendiendo a las necesidades agrícolas, y por ello la producción de la central tenía un carácter precario y dependiente de la voluntad de los regantes. La Central Térmica de Alicante era muy antigua, con motores en estado muy deficiente, lo que daba origen a ufrecuentes averías y largas y continuas reparaciones, por lo que no puede contarse, práctica y eficazmente, como fuente continua de producción» (17). Por otro lado, en lo que respecta a la energía importada, los suministros dependen de Hidroeléctrica Española que tiene que acoplar su distribución a extensas regiones, entre otras Madrid a la que suministra más del 50 por 100 de su consumo (18).

\section{CUADRO 1}

\section{EVOLUCIÓN DEL CONSUMO DE ENERGÍA ELÉCTRICA EN ESPAÑA Y EN LA PROVINCIA DE ALICANTE, 1942-1951}

\begin{tabular}{|c|c|c|c|c|}
\hline \multirow[t]{2}{*}{ AÑOS } & \multicolumn{2}{|c|}{ ALICANTE } & \multicolumn{2}{|c|}{ ESPAÑA (3) } \\
\hline & MILES KWH & $\overline{\text { ÍNDICE }}$ & MILLONES KWH & $\overline{\text { ÍNDICE }}$ \\
\hline 1942 & $147.323,7(1)$ & 100,0 & $4.437,6$ & 100,0 \\
\hline 1943 & $138.988,1(1)$ & 94,3 & $4.775,5$ & 107,6 \\
\hline 1944 & $105.188,1(1)$ & 71,4 & $5.719,5$ & 106,3 \\
\hline 1945 & $87.171,6(1)$ & 59,2 & $4.172,6$ & 94,0 \\
\hline 1946 & $147.678,1(2)$ & 100,2 & $5.411,3$ & 121,9 \\
\hline 1947 & $161.529,8(2)$ & 109,6 & $5.959,9$ & 134,1 \\
\hline 1948 & $161.813,8(2)$ & 109,8 & $6.111,0$ & 137,7 \\
\hline 1949 & $147.203,8(2)$ & 99,9 & $5.629,0$ & 126,8 \\
\hline 1950 & $164,885,2(2)$ & 111,9 & $6.916,0$ & 155,8 \\
\hline 1951 & $199.081,7(2)$ & 135,1 & $8.299,0$ & 187,0 \\
\hline
\end{tabular}

FUENTE: 1. Cámara Oficial de Comercio, Industria y Navegación de Alicante, Memoria Comercial 1944-5. 2. INE, Reseña estadística de la provincia de Alicante 1958. 3. INE, Anuario Estadístico de España, 1952. Elaboración propia.

(17) AHPA, Sección AISS, Legajo 2332-3a, Carpeta 4. ${ }^{\mathrm{a}}$.

(18) Idem. La Delegación Provincial de Industria señalaba, trasluciendo cierta insatisfacción, que «no puede exigirse a Hidroeléctrica que la producción de sus centrales en la zona de Levante la invierta en los propios lugares de producción. Ha de atender a toda su red, y se da el caso específico de que la producción del Jucar tiene que llevar a Madrid $500.000 \mathrm{kwh}$ diarios más o menos, sin cuya aportación extraprovincial el problema de Levante estaría no sólo muy aliviado sino resuelto». 
El resultado de esta situación es el que refleja el cuadro 1. El consumo provincial de electricidad permanece estancado hasta practicamente 1950 mientras el nacional presenta un ritmo de moderado ascenso a partir de 1946. Por otro lado, cuanto más intensas son las restricciones (trienio 1944-1946 y. 1949) mayor es el diferencial que se abre entre ambos índices, signo inequívoco de que la provincia de Alicante se vió más afectada por los cortes de fluido eléctrico. Por ende, los perjuicios económicos ocasionados por la escasez de ene rgía fueron más importantes en la provincia en tanto en cuanto las restricciones abortaron toda posibilidad de crecimiento, sobre todo en la industria pero también en el sector agrario, sin olvidar la repercusión entre la población activa, que vió aumentar el paro y disminuir sus ingresos.

\section{INFLUENCIA DE LA ESCASEZ DE ENERGÍA EN LA ECONOMIA ALICANTINA}

\section{a. La industria}

Las actividades industriales fueron las más perjudicadas por las restricciones eléctricas. Las repercusiones fueron «catastróficas, como no podía menos de suceder al reducirse a 15 ó 18 horas de trabajo semanal en la industria» (19). Esta extrema escasez ocurrió entre agosto de 1945 y febrero de 1946 (20), momentos de mayor estiaje; generalmente la intensidad mayor de los cortes se alcanzaba en el segundo semestre del año y, en función de las disponibilidades de los embalses y de la capacidad de producción eléctrica de origen térmico, se señalaban cortes que reducian el consumo a una media que oscilaba entre las 15 y 18 horas mencionadas, las 20 ó 25 de 1944, 1946 y 1949 (21) y las 30 horas de los restantes años.

Además del descenso de producción, la influencia de la escasez sobre la industria se traducia en el aplazamiento de la puesta en marcha de nuevas fábricas, ya que no se autorizaban instalaciones con energía eléctrica superior a 5HP, y en la prohibición de ampliación de las existentes. Todas las autorizaciones concedidas estaban condicionadas a la producción de energía propia con motores térmicos lo que llevó a la compra «a precios inverosimiles (hasta 1.500 pesetas por HP) de motores térmicos (de segunda mano y me-

(19) COCINA, op. cit.

(20) B.O.P. de 21 de agosto, 12 de septiembre, 26 de septiembre y 3 de octubre de 1945.

(21) B.O.P. de 5 de agosto, 5 de octubre y 20 de diciembre de 1944; 1 de octubre, 14 de octubre, 15 de noviembre, 18 de noviembre y 22 de diciembre de 1946; 6 de febrero y 10 de mayo de 1949. 
diano estado) de todo tipo a gas pobre, gasolina, gas-oil, diesel-oil y fuel-oil» (22). Estas máquinas, desde el punto de vista económico e incluso técnico, eran absurdas, sobre todo pensando que los suministros de carburantes tampoco se realizaban en la cantidad necesaria (23).

La falta de energía se constituia pues en una causa de estancamiento industrial más que agregar a las existentes en la postguerra que se concretaban en la carencia de materias primas, de bienes de equipo o en la contracción del mercado, y que a finales de década, una vez desaparecidas éstas últimas en gran medida, se convirtió en el factor principal de recesión. Un informe de la Cámara de Comercio de 1949 sobre la industria alicantina apuntaba:

«A las dificultades anteriormente señaladas (orografía) unimos las propias del momento, que se condensan en las restricciones eléctricas principalmente, ya que la cuestión de las materias primas y el auge de los mercados no supone ya serio problema» (24)

Conocer por todo ello la proporción justa en que los cortes eléctricos afectaron a la industria en la década de los cuarenta es dificultoso. Técnicamente la producción se reduce en la misma medida que las restricciones, pero en realidad, tal como indicaba el Delegado de Industria de Alicante:

"desciende en grado mayor por el menor rendimiento de las máquinas receptoras. Además la falta de frecuencia, sobre todo, ocasiona calentamientos excesivos en los motores, habiéndose ya inutilizados muchisimos, constituyendo este efecto una amenaza en potencia contra el utillaje motor de la provincia" (25)

Para evaluar dicha proporción deberiamos conocer la electricidad que se hubiera consumido en la industria sin restricciones lo que hubiese significado por otra parte la eliminación de la prohibición de ampliar o construir ins-

(22) COCINA, op. cit.

(23) Entre 1944 y 1945 los suministros «vienen siendo inferiores al 50 por 100 de las verdaderas necesidades» según Ministerio de Industria y Comercio, op. cit.. Por otro lado, en febrero de 1949 se establecieron los cupos de carburantes para las regiones con poca eléctricidad, claramente insuficientes (30-40 por 100 de las necesidades) para la provincia, lo que provocó el viaje del Gobernador Civil a Madrid para obtener cupos extraordinarios, concedidos en parte en el mes de junio de 1949 (vid. Información, 19 de febrero de 1949 y 24 de junio de 1949).

(24) COCINA, Memoria Comercial 1949, Alicante, 1960.

(25) Información, 24 de octubre de 1944. 
talaciones industriales. Para ello hemos elaborado el cuadro 2 donde aparece el consumo real de energía eléctrica en la provincia (destinado en un 5560 por 100 a la industria) y el hipotético, resultado de aplicar un incremento anual compuesto de un 10 por 100 sobre el consumo de 1942, año en el que no hubo cortes. Dicho incremento es el que se vino realizando desde que acabó la guerra hasta que se iniciaron las restricciones, contando con las dificultades ya señaladas de la industria del momento, según datós proporcionados por la Empresas suministradoras que recogen los informes económicos de estos años (26).

Tomando esta hipótesis de trabajo con las lógicas reservas, el resultado de contrastar ambas columnas arroja como principal conclusión la reducción en un 40 por 100 por término medio de la energía eléctrica consumida en la provincia, destacando por la importancia del descenso los años 1945, 1949

\section{CUADRO 2}

\section{EVOLUCIÓN DE LA PRODUCCIÓN, CONSUMO REAL Y CONSUMO HIPOTÉTICO DE ENERGÍA ELÉCTRICA EN LA PROVINCIA DE ALICANTE, 1942-1951 EN KWH}

\begin{tabular}{|c|c|c|c|c|c|c|}
\hline \multirow[t]{2}{*}{ AÑOS } & \multicolumn{3}{|c|}{ PRODUCCIÓN } & \multirow[t]{2}{*}{ CONSUMO REAL } & \multirow[t]{2}{*}{ C. HIPOTÉTICO * } & \multirow[t]{2}{*}{ \% C.r./C.h. } \\
\hline & HIDRÁULICA & TÉRMICA & TOTAL & & & \\
\hline 1942 & & & & 147.323 .661 & & \\
\hline 1943 & & & & 138.988 .117 & 162.056 .027 & $-14,23$ \\
\hline 1944 & 362.616 & 5.729 .516 & 6.092 .516 & 105.188 .117 & 178.361 .629 & $-41,02$ \\
\hline 1945 & $1.198: 000$ & 8.543 .000 & 19.741 .000 & 87.171 .566 & 196.197 .791 & $-55,56$ \\
\hline 1946 & 1.851 .000 & 9.857 .000 & 11.708 .000 & 147.678 .138 & 215.817 .570 & $-31,57$ \\
\hline 1947 & 2.966 .000 & 12.610 .000 & 15.576 .000 & 161.529 .818 & 237.399.327 & $-31,95$ \\
\hline 1948 & 3.064 .850 & 12.121 .992 & 15.186 .842 & 161.813 .820 & 261.139 .259 & $-38,03$ \\
\hline 1949 & 3.695 .100 & 24.312 .441 & 28.007 .541 & 147.203 .819 & 287.253 .284 & $-48,75$ \\
\hline 1950 & 2.830 .700 & 26.113 .259 & 28.943 .959 & 164.885 .252 & 315.978 .612 & $-47,81$ \\
\hline 1951 & 1.743 .800 & 17.068 .478 & 18.812 .278 & 199.081 .689 & 347.576 .473 & $-42,72$ \\
\hline
\end{tabular}

FUENTE: 1. COCINA, Memoria Comercial $1944-5$ e INE, Reseña estadística de la provincia de Alicante 1959; Elaboración propia.

* Consumo hipotético = Incremento anual compuesto del 10 por 100 sobre el consumo real de 1942.

(26) Ministerio de Industria y Comercio, op. cit., COCINA, Memoria Comercial 1944-5, y AHPA, Sección Aiss, Legajo 2332-3a, Carpeta 4. ${ }^{2}$, Ponencia «Fuerza motriz». 
y 1950 con decrementos de $55,56 \%, 48,75 \%$ y $47,81 \%$ respectivamente; porcentajes que debieron afectar, como se ha señalado, practicamente en la misma medida sobre la producción industrial provincial dada la exigua importancia que adquirió la energía alternativa como fuerza motriz en las fábricas.

Este descenso hipotético, provocado por el estancamiento en el suministro eléctrico, es complementario a su vez del realmente producido en las actividades fabriles. Analizando la producción de diferentes sectores industriales alicantinos en los que la fuerza motriz eléctrica es básica comprobamos esta incidencia (ver cuadro 3); el escaso crecimiento de la producción es evidente en todos ellos observandose descensos significativos fundamentalmente en 1949, año en el que la escasez eléctrica fue como hemos visto la única dificultad de trascendencia para la producción industrial, y en menor medida en 1945, excepto para la industria del calzado, actividad en la que el trienio 1944-6 fue el período más negativo (decremento de 5 puntos).

En similares circunstancias que la industria zapatera debió encontrarse la textil, también con importante peso dentro del sector secundario provincial. La elaboración de los índices de producción de esta industria es muy dificultosa teniendo en cuenta la gran cantidad de instalaciones y la diversidad de actividades que en ellas se desarrollan, lo que nos ha llevado a desechar las estadísticas disponibles. La repercusión de las restricciones debió ser importante si pensamos, por ejemplo, que la industria algodonera alcoyana, la segunda en importancia de España tras Barcelona, disponía como fuerza motriz de 2.565 C.V. en 1943 de los que 2.493 C.V. tenían origen eléctrico (27). No es extraño por ello que en los estudios monográficos realizados sobre esta industria que hacen referencia a nuestro período de estudio se señale como causas únicas de la crisis del sector la falta de materias primas y la escasez de energía eléctrica (28), factores que provocaron la recesión económica sin precedentes en l'Alcoía que conllevó incluso el descenso de su población absoluta.

(27) INE, Anuario Estadístico de España 1943.

(28) vid, por ejemplo, el artículo de Rosa María Jordá Borrell, «Alcoy: la crisis textil de 1965 y sus repercusiones» en Cuadernos de Geografía, número 18, pag. 29. 


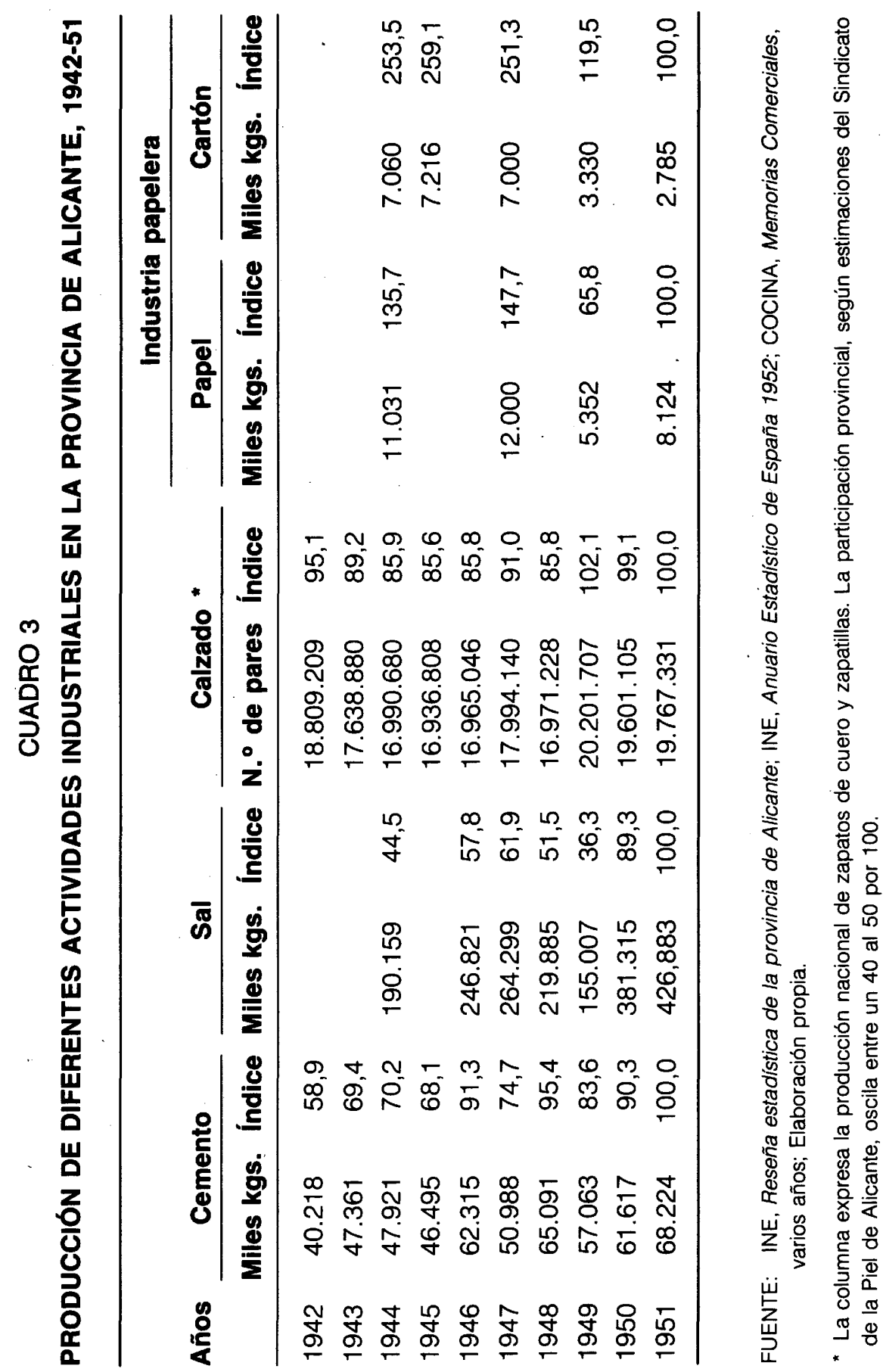




\section{b. La agricultura}

Las consecuencias de las restricciones en el campo fueron también importantes teniendo en cuenta que el riego en la provincia de Alicante se consigue en gran parte mediante la elevación del agua por bombas eléctricas. Ello sucede fundamentalmente en el oeste (Villena) con unas 3.500 hectáreas de cultivo de regadío gracias a las disponibilidades de agua subterranea, y en la zona central (Elche) cuyo sistema de riegos se basa en la utilizacion del caudal sobrante del río Segura en su desembocadura, otorgado en concesión a la Compañía Riegos de Levante, que eleva el agua por grupos motobombas eléctricos para abastecer a una amplia zona de 60.000 hectáreas distribuidas en 13 términos municipales a través de 225 kilómetros de canales (29). No obstante, las restricciones eléctricas nunca deben ser consideradas como causa básica del estancamiento o descenso de la producción agrícola durante los años cuarenta; la falta de agua en el agro era debida al estiaje y la carencia de energía se constituía en un factor más que venía a agravar la situación, puesto que suponía la reducción del numero de horas que el agricultor podía dedicar al riego de sus tierras insuficientemente regadas ya. En cambio, la falta de energía eléctrica si fue la causa directa por ejemplo del número escaso de obras destinadas a la explotación de pozos de riego entre 1945-50.

La disminución del número de horas de riego constituyó un grave problema en los años 1944-6 y 1949, coincidiendo con la mayor escasez de agua, toda vez que el resto de los años las restricciones se limitaron a las horas diurnas de los días laborables teniendo durante las noches y festivos suministro continuo, lo que suponía una merma de escasa consideración. Sin embargo, en los años indicados las disposiciones fueron mucho más severas; así el 5 de agosto de 1944 se determinaba la reducción del consumo al 50 por 100 en relación al mes de máximo consumo del año anterior; el 20 de diciembre se señalaba una reducción del 65 por 100 en relación al efectuado en análoga época de 1943; el 12 de septiembre de 1945, ante la agravación del problema, se restringe el consumo a 10 horas durante tres días laborables más 17 horas los domingos; en febrero de 1946 se elimina dicho recorte siendo sustituido por otro que contemplaba 9 horas de suministro nocturno durante cuatro o cinco días, situación que se mantuvo durante 1946 y que se volvió a repetir en 1949. (30)

Ahora bien, un problema latente a lo largo de toda la década era el de

(29) COCINA, Memoria comercial 1944-5, pag. 6.9.

(30) vid. B.O.P. de dichas fechas. 
la pérdida de eficacia de las bombas elevadoras. Tal como indica el Ingeniero Jefe de la Delegación de Industria de Alicante:

«La disminución de las horas de riego no refleja la verdadera situación más que de un modo aparente, pues las deficientes características de la corriente tanto en voltaje como frecuencia - de imposible mejora - se traducen en un aprovechamiento seminulo de las bombas elevadoras, que por girar a una velocidad inferior a su régimen normal de marcha, no elevan agua o lo hacen escasamente» (31)

De esta forma resultaba que las horas de riego eran realmente ilusorias. La ponencia «Aguas para Riegos» presentada en el I Consejo Económico Sindical Provincial de Alicante en 1946 era explícita en este sentido (32). Refiriéndose a las zonas regadas por la Compañía Riegos de Levante (las únicas de categoría provincial, las más modernas y extensas) señalaba como problema fundamental las dificultades ligadas a la restricción del fluido eléctrico, la falta de capacidad de las instalaciones, el bajo rendimiento de los grupos elevadores y la escasez de recambios.

El problema era más agudo si tenemos en cuenta que la causa básica de la falta de energía se centraba en lo manifiestamente insuficientes que resultaba la electricidad destinada a riegos. La Compañía Riegos de Levante se nutría del salto de Almadenes cuyos inconvenientes ya se han señalado. Esta central resultó en los primeros años de los cuarenta escasa entre otras razones porque alparecer la Compañía faltó a las condiciones fundamentales de la concesión, llevó agua a términos municipales no autorizados y modificó las condiciones del proyecto creando una zona regable enormemente superior a sus posibilidades hidráulicas (33). La solución pasaba por la construcción de centrales hidroeléctricas cuya producción debía ser dedicada con preferencia a fines agrícolas vinculando la energía necesaria a las elevaciones de «Riegos de Levante». La conclusión quinta de la citada ponencia recababa al Estado para

«que se acometa la ejecución de los aprovechamientos hidroeléctricos del Alto Segura reservando la energía necesaria para las atenciones de los riegos de la zona servida por las elevaciones de la Com-

(31) Información, 24 de octubre de 1944.

(32) AHPA, Sección AISS, Legajo 2332-3a, Carpeta 4. ${ }^{\mathrm{a}}$.

(33) Tal como señala la Orden de 13 de noviembre de 1940 que ordena la constitución de una Comunidad de Regantes y la intervención en la Compañía de un Tribunal de Aguas. 
pañia Riegos de Levante, al precio que fije la Administración, energía que quedaría vinculada a las existentes»

Pero las quejas del campesino alicantino iban dirigidas tanto al deficiente y escaso suministro eléctrico como a la escasez de maquinaria y materiales de conducción de corriente. En la reunión del sector Campo de la Organización Sindical Provincial celebrada en octubre de 1946 (34) se precisaba lo necesario que serían estos materiales para elevar la riqueza agrícola provincial. En concreto, la Comunidad de Riegos de Novelda, ante la escasez de agua para atender sus terrenos de regadío dedicados al tomate tardío y uva de mesa, inició unos trabajos de captación en el paraje del Salicejo en el término municipal de Villena. De los cinco pozos abiertos sólo dos podían ser utilizados por «la falta de cables de determinada sección para conducir más energía, al propio tiempo que le faltan también alguna máquina y ciertos útiles para el total montaje de las instalaciones que le precisan». Por otro lado, los 120 libros por $\mathrm{m}^{2}$ alumbrados por esos dos pozos, dado lo discontinuo del suministro eléctrico, no pueden ser elevados «durante un período ininterrumpido de tiempo". En Monforte del Cid, por otro lado, gracias a la iniciativa de los agricultores de municipios vecinos encabezados por Novelda, fue encontrada agua mediante perforaciones, se procedió a la construcción de pozos y balsas aunque «las instalaciones se retrasan porque no encuentran facilidades para adquirir aparatos y utillaje eléctrico con que dar fin a su loable proyecto».

A la vista de lo expuesto podemos señalar que la falta de energía eléctrica, la escasez de maquinaria unido a la característica falta de agua, imposibilitaron el aumento de los regadios en la provincia de Alicante a lo largo de los años cuarenta, fundamentalmente en aquellas zonas con dependencia eléctrica para el riego como el Campo de Alicante, el Valle del Vinalopó y, en menor medida, la Marina Alta. (35)

Un repaso a las extensiones de regadío dedicadas a los cultivos con mayor dedicación en el agro provincial refleja no obstante un aumento, si no importante, sí significativo. Entre los cereales la superficie de regadío dedicada al trigo en 1945 era de 9.530 has mientras en 1950 era de 11.996 (crecimiento de 25,8 por 100 ); la superficie dedicada a la cebada pasa de 7.731 has en 1945 a 8.360 en 1950 (incremento de 8,1 por 100); el arroz era sembrado

(34) AHPA, Sección AISS, Legajo 2332-3a, Carpeta 3. ${ }^{\text {. }}$

(35) A todo ello hay que añadir el casi total abandono que sufrió la provincia de Alicante por parte del Instituto Nacional de Colonización, tal como destaca J. Sorní Mañés, «La política agraria en el País Valenciano (1939-75)» en Agricultura y Sociedad, número 16, 1980. 
en 588 has en 1945 mientras en 1950 lo era en 1.275 has (ascenso de 116,8 por 100). Lo mismo ocurre entre las leguminosas (las judias y las habas pasan de poseer una extensión de 236 y 1325 has en 1945 a 280 y 1850 en 1950 respectivamente, lo que supone aumentos de 18,6 y 39,6 por 100), la patata (de 3.400 a 4.465 con un crecimiento de 31,3 por 100), la vid (de 45.711 a 46.286 -ascenso de 1,2 por 100-y los cultivos hortícolas (la alcachofa por ejemplo pasa de 632 has a 650 has con un aumento de 2,8 por 100) (36).

Sin embargo, todos estos aumentos deben ser tomados con precaución pues, tal como indica el Consejo Económico Sindical Provincial de Alicante, el crecimiento teórico del regadío provincial entre 1940 y 1959 era sólo ficticio y la superficie regada realmente oscilaba entre $1 / 6$ y $1 / 7$ de la señalada (37). La coyuntura de escasez de maquinaria y energía tuvo que provocar un lento desarrollo de las obras destinadas a extender el regadío provincial. Un buen índice para medir este aspecto nos lo proporciona el cuadro 4 en el que se especifica el número de autorizaciones concedidas para la explotación de pozos de riego entre 1946 y 1956. La elevación del agua de estos pozos se hacía fundamentalmente mediante la utilización de motores eléctricos en una proporción para los dos períodos confeccionados de cuatro motores por cada cinco usados. La comparación entre las dos etapas nos permite establecer las siguientes conclusiones:

- el número de autorizaciones se multiplica en el primer quinquenio de la década de los cincuenta con respecto al período anterior por 2,5.

- la proporción de la potencia eléctrica instalada respecto a la total pasa de 75,1 por 100 en la primera etapa a 77,7 por 100 en la segunda. Por otro lado, esta potencia es 1,89 veces superior en 1951-6 que en el segundo quinquenio de los años cuarenta.

- la capacidad total de aguas elevadas es practicamente el doble en 1951-6 en relación a 1946-50.

En definitiva, considerando el segundo período como normal, las obras de elevación de aguas, básicas en la infraestructura del regadío provincial, realizadas en el período de escasez energética tan sólo fueron la mitad de las que sebían haber realizado, lo que significó una disposición de aguas muy reducidas.

(36) Datos extraidos de los Anuarios Estadísticos de España de 1946 y 1951.

(37) Consejo Económico Sindical Provincial de Alicante, Estructura y posibilidades de desarrollo económico de Alicante, Alicante, 1959. pags. 100-103. 
CUADRO 4

AUTORIZACIONES PARA EXPLOTACIÓN DE POZOS DE RIEGO 1946 - 1956

\begin{tabular}{|c|c|c|c|c|c|c|}
\hline \multirow[b]{2}{*}{ ANNOS } & \multirow{2}{*}{$\begin{array}{c}\text { NÚMERO } \\
\text { DE } \\
\text { AUTS. }\end{array}$} & \multicolumn{4}{|c|}{$\begin{array}{l}\text { POTENCIA INSTALADA EN DICHAS } \\
\text { CONCESIONES (C.V.) }\end{array}$} & \multirow{2}{*}{$\begin{array}{c}\text { CAPACIDAD TOTAL } \\
\text { DE ELEVACIÓN } \\
\text { DE AGUAS } \\
\text { LITROS POR MINUTO }\end{array}$} \\
\hline & & ELÉCTRICA & GASOLINA & GASOIL & TOTAL & \\
\hline 1946 & 39 & 517 & 126 & 20 & 663 & 56.060 \\
\hline 1947 & 20 & 354 & 177 & 140 & 671 & 14.020 \\
\hline 1948 & 26 & 1.093 & 50 & 199 & 1.342 & 71.400 \\
\hline 1949 & 19 & 447 & 31 & 118 & 596 & 25.300 \\
\hline 1950 & 18 & 451 & 50 & 36 & 537 & 31.800 \\
\hline \multicolumn{7}{|c|}{ Promedio anual } \\
\hline $1946-1950$ & 24,4 & 572,4 & 86,8 & 102,6 & 761,8 & 39.716 \\
\hline 1951 & 59 & 1.604 & 77 & 110 & 1.791 & 90.350 \\
\hline 1952 & 65 & 797 & 97 & 243 & 1.137 & 44.200 \\
\hline 1953 & 69 & 1.211 & 56 & 257 & 1.524 & 98.800 \\
\hline 1954 & 64 & 402 & 91 & 174 & 667 & 41.000 \\
\hline 1955 & 40 & 1.141 & 21 & 91 & 1.253 & 78.000 \\
\hline 1956 & 64 & 1.321 & 57 & 590 & 1.968 & 82.000 \\
\hline \multicolumn{7}{|c|}{ Promedio anual } \\
\hline $1951-1956$ & 60,1 & $1.079,3$ & 66,5 & 244,1 & 1.390 & $72.391,6$ \\
\hline
\end{tabular}

FUENTE: INE, Reseña Estadística de la provincia de Alicante 1958, Alicante, 1959. Elaboración propia.

\section{c. El paro}

La faceta más intranquilizadora del problema energético se planteaba en el aspecto social y se concretaba en el pago de los jornales correspondientes a los días de restricción. Más que de paro podemos hablar, por tanto, de desocupación y del grave problema que ésta conllevaba por lo que significaba de reducción de salario. Esta circunstancia se iniciaba en el momento en el que las jornadas de trabajo perdidas eran irrecuperables debido a la imposibilidad de trabajar más de ocho horas diarias en los días con suministro de corriente o los festivos. Ello ocurre por primera vez de manera importante y sostenida el mes de agosto de 1944 (38), fecha en la que el Delegado Técni-

(38) BOP del 5 de agosto de 1944. 
co de la zona de Levante dispone el corte se suministró tres días a la semana y la imposibilidad de recuperarlos. La Disposición cuarta precisaba:

"La prohición de consumir energía eléctrica en los días señalados es absoluta, entendiéndose que no se podrá recuperar ninguna de las horas perdidas ni siquiera en los domingos».

El problema laboral originado por tal disposición necesitaba una solución que a ojos del obrero y del empresario fuese lo más equitativa posible. El Gobernador civil, tras consulta con la Delegación de Trabajo, obligó al empresario a satisfacer una de las tres jornadas perdidas sin compensación alguna, a abonar otra con derecho a descontarla de las vacaciones anuales retribuidas dejando por otro lado de ser percibida la tercera jornada por el trabajador «que de esta forma se solidariza con las desagradables consecuencias que imponen las adversas circunstancias presentes» (39). Al agotarse por perdidas el período de vacaciones retribuidas en octubre se hizo necesario modificar las normas anteriores puesto que carecían de base. La Delegación Provincial de Trabajo acordó que, con caracter obligatorio, la Empresa abonase la mitad del tiempo de paralización de las actividades por falta de energía eléctrica, mientras el trabajador debía recuperar cuando el suministro lo permitiese la mitad del tiempo abonado (es decir, cuatro horas en tanto la paralización sea de dos días y seis horas en caso de que fuesen tres días) (40), por lo que el asalariado veía afectadas $3 / 4$ partes de su jornal correspondiente a los días de restricción.

Esta circunstancia, unida a la impresión de «parcheo» que resultaba de esta política, hizo necesaria la redacción de una Disposición General sobre la materia. Por otro lado, la extremada sequía que se padeció entre 1944-5 y que hacía preveer una restricción aún mayor de energía en el segundo semestre de 1945 (41) hacia impracticables las disposiciones locales por cuanto suponian el desgaste total de los beneficios acumulados. De esta forma surge el Decreto-Ley de 3 de agosto de 1945 sobre «subsidios al personal obrero afectado por el Paro originado por la escasez de suministro de energía eléctrica» (42). Su objetivo era:

(39) BOP del 23 de agosto de 1944.

(40) BOP del 7 de octubre de 1944.

(41) Durante los meses de mayo y junio de 1945 de manera periódica se reproducía en la prensa un llamamiento «dramático» del Gobernador Civil a la «conciencia ciudadana» para evitar «una situación caótica que perturbaria seriamente nuestra economía» y que hacía presagiar la implantación de los cortes durante cuatro días tal como sucedió.

(42) Boletín Oficial del Estado, 9 de agosto de 1945. 
«aminorar un aspecto característico de la crisis existente procurando distribuir los daños, ya que así lo aconseja la extraordinaria anormalidad de las circunstancias que, por su origen y agudeza, no pueden ser soportados sin daños de mayor entidad por los sectores directamente afectados»

Según el Decreto, el obrero desocupado por la restricción tendrá derecho a percibir semanalmente las 5/6 partes de los jornales correspondientes a la semana normal de 48 horas cualquiera que sea el número de horas trabajadas (artículo 2). Las diferencias entre las cantidades que deban ser pagadas a los obreros y las que se deduzcan del pago de las horas realmente trabajadas en la semana serán adelantadas por las Empresas; las 2/3 partes de las cantidades así anticipadas serán satisfechas al empresario por la «Caja de Compensación del Paro por escasez de energía eléctrica» que este DecretoLey crea; la tercera parte restante será reintegrada por los obreros afectados en futuras horas extraordinarias (artículo 3). Con esta serie de disposiciones el trabajador, si bien veía disminuir su salario en parte, no se convertía en el principal perjudicado del paro industrial. Por otro lado, las empresas se veían libres al parecer del pago de unos jornales no trabajados encargándose el Estado, a través de la Caja de Compensación creada, de este cometido. Pero lógicamente esta Obra asistencial debía nutrirse de unos fondos que a la postre iban a ser costeados por el empresario, al menos en un alto porcentaje. Efectivamente, el artículo ocho preveía que el capital manejado por la $\mathrm{Ca}$ ja procedería de un recargo especial sobre el suministro de energía eléctrica que quedaba concretado en el 20 por 100 sobre las facturas de alumbrado y el 10 por 100 sobre las de fuerza (43). Como quiera que dicho recargo no admitía exención, practicamente la totalidad de las empresas afectadas se adhirieron al sistema, a pesar de que el Decreto contemplaba la posibilidad de que cada empresa arbitrase el suyo siempre y cuando fuese tan «equitativon como la Ley.

Lógicamente, con esta legislación quedó desamparada toda la población activa que trabajaba clandestinamente en sus respectivas casas. El alto índice de trabajo a domicilio que tradicionalmente ha caracterizado a la economía alicantina, debido al tipo de actividades que en la provincia se desarrolla -textiles, calzado, juguetes ...-, hace pensar que esta circunstancia fue más trascendente al sur del País Valenciano que, en general, en el conjunto de

(43) Dicho recargo fue precisado por Orden del Ministerio de Trabajo de 22 de octubre de 1945, BOP, 29 de octubre de 1945. 
la economía española. Baste citar, en este sentido, que de los 30.000 trabajadores del calzado existentes en la provincia en la década de los cuarenta, sólo unos 9.000 (8.938 exactamente) trabajaban en la legalidad (44).

Tampoco afectaba el Decreto-Ley a aquellos trabajadores que acabaron por perder su puesto de trabajo. El análisis de las cifras de paro del decenio posterior a la Guerra Civil señala un paralelismo significativo entre el recrudecimiento de las restricciones y aumento del paro. Las estadísticas de población parada recogidas son las proporcionadas por la Oficina Provincial de Encuadramiento y Colocación Obrera de Alicante (45), que no adjuntan la población economicamente activa total de cada momento si bien son perfectamente validas toda vez que la población alicantina apenas sufre modificaciones de importancia durante el período analizado. El gráfico II, elaborado a partir de estas cifras, nos muestra como el descenso ininterrumpido del paro en 1943 (tendencia que se venía sosteniendo desde que terminó la guerra) se rompe en 1944 ligeramente. La disminución brusca del primer semestre de 1945 da paso a un período de año y medio de ascenso fuerte del paro alicantino, seguido de una nueva baja entre 1947 y 1948 para terminar el decenio con un aumento importante de la cifra de parados. La correlación nitida entre fuertes restricciones y aumento del paro explica la influencia del primer factor sobre el segundo, incidencia que debió ser mayor que la recogida en las estadísticas oficiales. Los cortes de fluido eléctrico en la industria constituían pues un problema más que agregar a las muchas dificultades que este sector económico tenía en la postguerra que se concretaban fundamentalmente en la escasez de materias primas. Así lo expresaba un informe sobre el año 1946 de la Oficina de Colocación:

«Respecto de la industria textil ocurre también que la falta de algodón por dificultades de importación, y la restricción de fluido eléctri$\mathrm{co}$, hacen que se mantenga el paro. Otro tanto acontece con la de la piel, por la escasez de cueros y piel, así como de clavazón, al paso que, como antes, las restricciones agravan más su situación.» (46)

(44) Según el Sindicato Provincial de la Piel en la ponencia «Problemas del calzado» presentada en el I Consejo Económico Sindical Provincial en 1946.

(45) Reproducidas por los Anuarios Estadísticos de España y las Memorias Comerciales de la COCINA. Hay que tomarlas con la lógica reserva puesto que no contabilizaban como parados aquellos trabajadores que aunque sea sólo temporalmente se dedicaban a labores de Obras Públicas emprendidas por el Estado y la Diputación con el fin de remediar el paro.

(46) Cocina, Memoria Comercial 1946, Alicante, 1947. 


\section{GRÁFICO I \\ EVOLUCIÓN DE LA POBLACIÓN PARADA EN LA PROVINCIA \\ DE ALICANTE, 1942 - 1951}

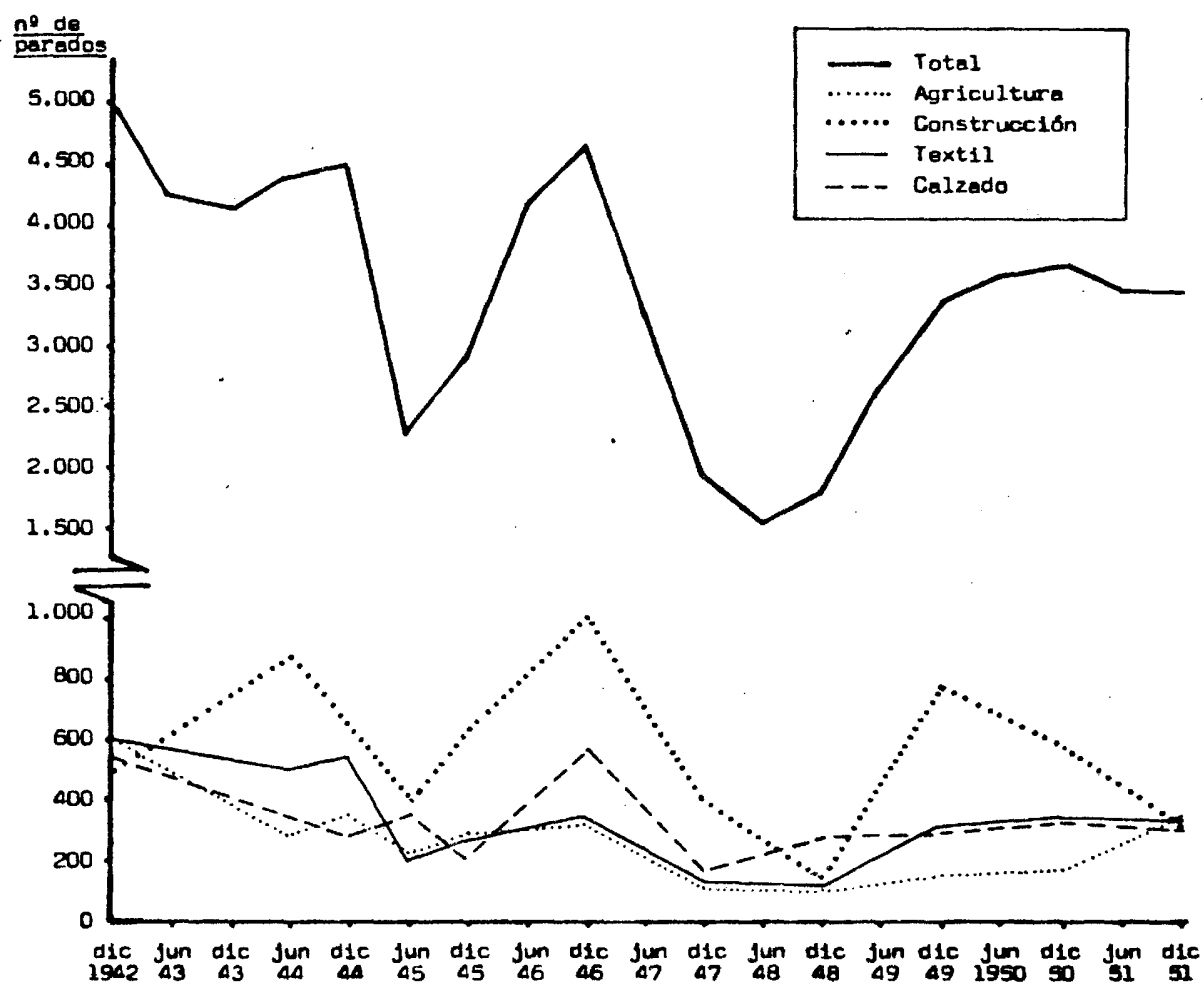

FUENTE: COCIN, Memorias Comerciales e INE, Anuarios estadísticos 
La opinión del momento era más explicita aún y fijaba en la falta de energía la principal causa del problema. Textos como el siguiente eran corrientes en. la prensa:

«Pero de improviso se produce ... una de las sequias más terribles que se han producido en nuestra Patria: las labores tanto industriales como agrícolas sufrieron el descenso que todos conocemos y el paro obrero se presentó inmediatamente como un peligro capaz de dar al traste con una labor que ... era orgullo del Nuevo Estado» (47)

El análisis de las actividades económicas con mayor número de parados en la provincia refleja como éstas presentan un comportamiento similar al visto. La construcción, sector indicativo siempre de la evolución económica, se muestra más afectado como consecuencia, por un lado, de la contracción general, y por otro, por la carencia de materiales dada la dificultad de adquisición de hierro y cemento acentuada en los años de mayores cortes de energía, que obligaba a paralizar temporal o definitivamente obras en ejecución o impide que gran número de proyectos sean acometidos. El calzado y la industria textil, las dos actividades industriales más importantes en la provincia, presentan un menor número de parados aunque éste, dado el alto porcentaje de obreros no censados en ambos sectores, debió ser mayor. La recesión es más clara si tenemos en cuenta que de cerca de 9.000 obreros del calzado existentes en 1944-5 se pasa a tan solo 8.069 en 1947 (48), lo que supone una reducción de 900 obreros, cifra cinco veces superior a las estadisticas de paro oficiales dadas para el sector en 1947. En este mismo sentido cabe hablar de la agricultura, por la característica idiosincracia de este sector económico, que presenta no obstante una evolución semejante.

(47) Información, 1 de enero de 1946.

(48) Ibidem nota 44. 